\section{BMJ Open Respiratory Research}

\title{
Characteristics and outcomes of admitted patients infected with SARS- CoV-2 in Uganda
}

Bruce Kirenga (D) , ${ }^{1}$ Winters Muttamba, ${ }^{1}$ Alex Kayongo, ${ }^{1}$ Christopher Nsereko, ${ }^{2}$ Trishul Siddharthan, ${ }^{3}$ John Lusiba, ${ }^{4}$ Levicatus Mugenyi, ${ }^{1}$ Rosemary K Byanyima, ${ }^{5}$ William Worodria, ${ }^{6}$ Fred Nakwagala, ${ }^{7}$ Rebecca Nantanda, ${ }^{1}$ Ivan Kimuli, ${ }^{1}$ Winceslaus Katagira, ${ }^{1}$ Bernard Sentalo Bagaya, ${ }^{8}$ Emmanuel Nasinghe, ${ }^{8}$ Hellen Aanyu-Tukamuhebwa, ${ }^{9}$ Beatrice Amuge, ${ }^{10}$ Rogers Sekibira, ${ }^{1}$ Esther Buregyeya, ${ }^{11}$ Noah Kiwanuka, ${ }^{11}$ Moses Muwanga, ${ }^{12}$ Samuel Kalungi, ${ }^{13}$ Moses Lutaakome Joloba, ${ }^{8}$ David Patrick Kateete, ${ }^{8}$ Baterana Byarugaba, ${ }^{7}$ Moses R Kamya, ${ }^{14}$ Henry Mwebesa, ${ }^{15}$ William Bazeyo ${ }^{16}$

To cite: Kirenga B, Muttamba W, Kayongo A, et al. Characteristics and outcomes of admitted patients infected with SARS-CoV-2 in Uganda. BMJ Open Resp Res 2020;7:e000646. doi:10.1136/ bmjresp-2020-000646

- Additional material is published online only. To view, please visit the journal online (http://dx.doi.org/10. 1136/bmjresp-2020-000646)

Received 21 May 2020 Revised 24 August 2020 Accepted 25 August 2020
Check for updates

(C) Author(s) (or their employer(s)) 2020. Re-use permitted under CC BY-NC. No commercial re-use. See rights and permissions. Published by BMJ.

For numbered affiliations see end of article.

Correspondence to Dr Bruce Kirenga; brucekirenga@yahoo.co.uk

\section{ABSTRACT}

Rationale Detailed data on the characteristics and outcomes of patients with COVID-19 in sub-Saharan Africa are limited.

Objective We determined the clinical characteristics and treatment outcomes of patients diagnosed with COVID-19 in Uganda.

Measurements As of the 16 May 2020, a total of 203 cases had been confirmed. We report on the first 56 patients; 29 received hydroxychloroquine $(\mathrm{HCQ})$ and 27 did not. Endpoints included admission to intensive care, mechanical ventilation or death during hospitalisation. Main results The median age was 34.2 years; $67.9 \%$ were male; and $14.6 \%$ were $<18$ years. Up $57.1 \%$ of the patients were asymptomatic. The most common symptoms were fever $(21.4 \%)$, cough (19.6\%), rhinorrhea $(16.1 \%)$, headache $(12.5 \%)$, muscle ache $(7.1 \%)$ and fatigue (7.1\%). Rates of comorbidities were $10.7 \%$ (pre-existing hypertension), 10.7\% (diabetes) and 7.1\% (HIV), Body Mass Index (BMl) of $\geq 3036.6 \% .37 .0 \%$ had a blood pressure (BP) of $>130 / 90 \mathrm{~mm} \mathrm{Hg}$, and $27.8 \%$ had BP of $>140 / 90 \mathrm{~mm} \mathrm{Hg}$. Laboratory derangements were leucopenia (10.6\%), lymphopenia (11.1\%) and thrombocytopenia (26.3\%). Abnormal chest X-ray was observed in $14.3 \%$. No patients reached the primary endpoint. Time to clinical recovery was shorter among patients who received $\mathrm{HCQ}$, but this difference did not reach statistical significance.

Conclusion Most of the patients with COVID-19 presented with mild disease and exhibited a clinical trajectory not similar to other countries. Outcomes did not differ by HCQ treatment status in line with other concluded studies on the benefit of using HCQ in the treatment of COVID-19.

\section{INTRODUCTION}

COVID-19, a disease caused by the SARS-CoV-2 virus, was first reported in early December 2019 in Wuhan China. ${ }^{1}$ The number of patients confirmed to have the disease rapidly increased and on 30 January

\section{Key messages}

Whereas COVID-19 is associated with high mortality, with a global mortality rate of $6.8 \%$, with about $13.8 \%$ of patients developing severe disease and $6 \%$ developing critical illness, the disease has not been well characterised in sub-saharan African countries.

Most of the initial patients with COVID-19 in Uganda presented with mild disease and exhibit a clinical trajectory not similar to other countries.

2020, and the disease was declared a public health emergency of international concern by the WHO. As of 16 May 2020, a total of 4396392 confirmed cases and 300441 deaths $(6.8 \%)$ had been reported globally. ${ }^{2}$ In Africa, 75498 cases and $2561(3.4 \%)$ deaths had been reported by 16 May 2020. ${ }^{3}$ Africa faces many well-documented barriers to addressing pandemics, and COVID-19 has the potential to strain existing health infrastructure.

The initial presentation of COVID-19 was of an acute respiratory illness, with many cases being diagnosed with pneumonia which rapidly progressed into respiratory failure. ${ }^{4}$ Symptoms encountered among confirmed patients include cough, fever, myalgia, fatigue, abdominal pain, diarrhoea, nausea, vomiting, headache and dyspnoea. ${ }^{4}$ It is now established that the majority of persons infected with SARS-CoV-2 virus are asymptomatic. A study of 215 pregnant mothers universally tested in New York found that $84.6 \%$ were negative, $13.5 \%$ positive and asymptomatic and only $1.9 \%$ were positive and symptomatic. $^{5}$ Patients with COVID-19 are characterised by the presence of comorbidities with 
diabetes, hypertension, asthma, chronic obstructive pulmonary disease (COPD), chronic kidney disease and a range of heart diseases being among the most encountered comorbidities. ${ }^{467}$ Several laboratory and imaging derangements, such as leucopenia, thrombocytopenia, raised d-dimers, C reactive protein (CRP) and high cytokine levels, have been reported among patients with COVID-19. ${ }^{4}$ By presentation, majority of patients have radiological changes on chest radiograph; commonly bilateral infiltrates and ground-glass opacities (GGO) are seen on chest CT scan images. ${ }^{467}$

COVID-19 is a fatal disease, and globally the mortality rate is $6.8 \% .^{2}$ It is estimated that about $13.8 \%$ of patients with COVID-19 develop severe disease, and 6\% develop critical illness defined as respiratory failure, shock and multiple organ dysfunction or failure, and dyspnoea, respiratory rate of $\geq 30$ breaths/min, oxygen saturation of $\leq 93 \%$, and partial pressure of arterial oxygen to fraction of inspired oxygen ratio $50 \%$ within $24-48$ hours, respectively. $^{89}$

Currently, there are no proven treatments and vaccines for COVID-19. Several repurposed and new drugs have been reported in compassionate use and small trials to offer some benefit. ${ }^{9}$ These drugs include the antimalarials (chloroquine and hydroxyl chloroquine), antivirals such as remdesivir and favipiravir, and antiretroviral combination therapies such as lopinavir/ritonavir. ${ }^{9-13}$ Anti-inflammatory drugs such as tocilizumab have been reported to have some effects. ${ }^{14}$ Convalescent plasma and ivermectin have additionally been trialled. ${ }^{11}{ }^{15}$ Globally, publications on the characteristics, outcomes and efficacy of candidate medications for COVID-19 are emerging, helping clinicians, public health scientists and political leaders frame appropriate epidemic control responses. In Uganda and most of Africa, COVID-19 literature is limited. This is probably because the disease has recently arrived in Africa but could also be due to limited capacity to simultaneously respond to the epidemic while collecting research publishable data at the same time. Uganda reported the first case of COVID-19 on 21 March 2020. Two treatment centres were established at Mulago National Specialised Hospital and Entebbe Regional Referral Hospital. We quickly formed a research group called COVIDRES-Uganda. The group is a multidisciplinary research group consisting of clinical, public health, behavioural and biomedical scientists. This group allowed the conduct of research alongside clinical care. The purpose of this paper was to describe the characteristics and treatment outcomes of the first patients with COVID-19 admitted at the two hospitals. We further compare the treatment outcomes by hospitals since one hospital provided hydroxychloroquine (HCQ) and the other did not.

\section{METHODS}

\section{Design, participants and study sites}

This study is a prospective cohort study. Patients diagnosed with confirmed COVID-19 in Uganda are all eligible to participate. The only exclusion criterion is failure to provide written informed consent/assent. Patients were consecutively enrolled and followed up until the first occurrence of one of the outcomes of discharge, transfer to intensive care unit (ICU), mechanical ventilation or death. In this paper, we describe only patients hospitalised at Mulago National Specialised Hospital and Entebbe Regional Referral Hospital, the largest COVID-19 treatment facilities in the country. Mulago National Specialised Hospital is a tertiary health facility with about 900 admission beds and 36 adult ICU beds located in the capital city of Uganda, Kampala. At the time of the outbreak, the hospital was undergoing a major renovation. However, one block, block A, had been completed. The entire block was made ready and converted into a COVID-19 screening (levels 2 and 3) and treatment centre (levels 4,5 and 6). At the time of this article, only level 4 was being used as a treatment centre due to the limited number of patients. Entebbe Regional Referral Hospital is located in Entebbe city at the shores of Lake Victoria. Entebbe city is home to the only international airport in Uganda, the Entebbe International Airport. It is a 200-bed referral hospital and has four ICU beds.

\section{Patient management \\ Clinical reviews}

At the time the first cases of COVID-19 were diagnosed in the country, there was no standardised case management protocol for the country. Sites managed patients based on individual specialists' advice and basing on international management guidelines, notably those published by WHO. ${ }^{16}$ For this reason, the management of patients differed in many ways at the two hospitals. At Mulago National Specialised Hospital, patients were managed in an open spaceward of 55 beds in total separated in cubicles of 4 beds each. The ward has four private rooms, three of which were occupied by patients. Adjacent to the ward, there was an office space that was used by health workers for donning personal protection equipment (PPE) and storing supplies. At Entebbe Regional Referral Hospital, similar open space wards were used. Health worker teams were multidisciplinary, consisting of specialist internal medicine physicians, a paediatrician, general practitioners, nurses, pharmacists, radiologists, pathologists, microbiologists, psychologists and psychiatrists. At Entebbe Regional Referral Hospital, similar composition of the team was used, but the team was smaller as some specialists are not part of the staffing norm of a regional referral hospital. PPE was used all the time and consisted of N95 masks, coveralls, aprons, gaggles, gumboots and gloves. Wards were periodically sanitised and hand sanitisers were used between patients. Patients were reviewed 
by specialist physicians once a day and by nurses and general physicians three times a day, that is, day shift, evening shift and night shift. At each review, saturation of peripheral oxygen $\left(\mathrm{SpO}_{2}\right)$, pulse rate $(\mathrm{PR})$, temperature, blood pressure (BP) and symptoms were charted. Other clinical assessments were performed as clinically relevant. Patients were discharged if they were asymptomatic, had completed 14 days of hospitalisation and had two negative reverse transcriptase PCR (RT-PCR) tests at least 24 hours apart.

\section{Laboratory and radiological examinations}

We performed baseline chest X-ray (CXR), ECG and laboratory tests. Baseline laboratory tests included complete blood count, liver and kidney function tests, CRP, d-dimers and high-sensitivity troponin. In addition to the clinical laboratory tests, consenting patients had the following samples obtained for biobanking: saliva, stool, blood, and nasal and oropharyngeal swabs. Swabs and blood sampling were repeated weekly until discharge. Clinical laboratory tests were repeated if indicated. One patient underwent CT scanning; one had blood culture; and patients with diabetes had blood sugar monitoring and glyacaeted haemoglobin (HbAlc) testing. At Entebbe Regional Referral Hospital, some laboratory tests were not performed and CXR was not done due to logistical constraints.

\section{Medications}

Medications administered differed between sites. At the Entebbe Regional Referral Hospital site, HCQ was administered for patients who were symptomatic, while at the Mulago National Specialised Hospital site it was not administered. Patients who had respiratory symptoms had a combination of ampiclox (a generic ampicillin/ cloxacillin combination) or augmentin plus azithromycin. Those with radiological evidence of lung involvement had the option of receiving intravenous ceftriazone plus azithromycin. All patients received vitamin $\mathrm{C}$ tabs supplementation. Comorbidities were treated as indicated.

\section{Definitions}

Fever was defined as a temporal temperature of $37.5^{\circ} \mathrm{C}$ or higher, leucopenia as total white cell count of $\leq 4000$ / $\mathrm{mL}^{3}$ and lymphocytopenia as a lymphocyte count of less than 1500 cells $/ \mathrm{mm}^{3}$. Thrombocytopenia was defined as a platelet count of less than $150000 / \mathrm{mm}^{3}$. Anaemia as haemoglobin level of $\leq 10 \mathrm{~g} / \mathrm{dL}$, high aspartate aminotransferase (AST) as AST $>40 \mathrm{U} / \mathrm{L}$, high alanine aminotransferase (ALT) as ALT $>40 \mathrm{U} / \mathrm{L}$, high creatinine as creatinine $\geq 133 \mu \mathrm{mol} / \mathrm{L}$, high lactate dehydrogenase $(\mathrm{LDH})$ as $\mathrm{LDH} \geq 250 \mathrm{U} / \mathrm{L}$, high troponin as troponin $\geq 0.06 \mathrm{ng} / \mathrm{mL}$, high total bilirubin as total bilirubin $>17.1$, high CRP as CRP $\geq 10 \mathrm{mg} / \mathrm{L}$ and high d-dimers as d-dimer levels of $\geq 0.5 \mathrm{mg} / \mathrm{L}$. Asymptomatic COVID-19 was defined as the absence of cough, fever (reported), temperature of $\geq 37.5^{\circ} \mathrm{C}$, rhinorrhea, fatigue and shortness of breath.

\section{Statistical analysis}

We used descriptive statistics including proportions, means, and medians to describe the patients' characteristics stratified by the two study sites; Mulago National Specialised Hospital and Entebbe Regional Referral Hospital. Chi-square or Fisher's exact tests were used to compare proportions, t-test to compare means, and Wilcoxon rank-sum test to compare medians between study sites. We used graphical aids to visualise trend in various symptoms and vital signs observed over a period of 14 days stratified by study sites. Survival analysis using Kaplan-Meier curves was used to estimate recovery rates from major symptoms of COVID-19 observed over the first 14 days. Log-rank test was used to compare recovery rates between sites. We also analysed for factors associated with asymptomatic infection of COVID-19 and we used OR with $95 \%$ CIs to quantify the associations. All analyses were done using STATA V.15.

\section{Patient and public involvement statement}

Given the strict national lockdown measures put in place due to the COVID-19 pandemic, we did not involve patients in the design of the study. Instead, through the Ministry of Health COVID-19 task Force, the public was informed about the recruitment and conduct of the study. Through the Ministry of Health public broadcasts and presidential COVID-19 updates, the general public has been informed about the study findings.

\section{RESULTS}

\section{Sociodemographic characteristics}

The baseline characteristics of the first 56 patients treated at Mulago National Specialised Hospital and Entebbe Regional Referral Hospital are presented in table 1 . Of the 56 patients, 25 were treated at Mulago National Specialised Hospital and 31 were treated at Entebbe Regional Referral Hospital. The mean age of all the patients was 34.2 years with an SD of 15.5 years; Mulago National Specialised Hospital versus Entebbe Regional Referral Hospital (42.2 vs 27, $\mathrm{p}=0.0003$ ). The youngest patient was 9 months while the oldest was 66 years. A total of $8(14.6 \%)$ patients were under the age of 18 years. By gender, $67.9 \%$ of the patients were male. Only one patient reported a history of tobacco smoking.

\section{Symptoms and comorbidities}

Patients who had any of the major COVID-19 symptoms defined as the presence of fever (reported), temperature of $\geq 37.5^{\circ} \mathrm{C}$, cough, shortness of breath, fatigue or rhinorrhea constituted $42.9 \%$ (24 patients). The most common symptoms were fever $(21.4 \%)$, cough $(19.6 \%)$, rhinorrhea $(16.1 \%)$, headache $(12.5 \%)$, muscle ache $(7.1 \%)$ and fatigue $(7.1 \%)$. No patient reported shortness of 
Table 1 Baseline characteristics of patients infected with SARS-CoV-2 admitted to two hospitals in Uganda

\begin{tabular}{|c|c|c|c|c|}
\hline Characteristic & All & $\begin{array}{l}\text { Mulago National } \\
\text { Specialised Hospital }\end{array}$ & $\begin{array}{l}\text { Entebbe Regional } \\
\text { Referral Hospital }\end{array}$ & $P$ value \\
\hline Enrolment hospital, $\mathrm{N}$ & 56 & 25 & 31 & \\
\hline Age (years) & $\mathrm{n}=55$ & $n=25$ & $n=30$ & \\
\hline Mean (SD) & $34.2(15.5)$ & $42.2(15.2)$ & $27.6(12.5)$ & $0.0003^{*}$ \\
\hline Median (IQR) & $33(25-43)$ & $42(33-54)$ & 27 (22-35) & $0.0004 \dagger$ \\
\hline \multicolumn{5}{|l|}{ Categorical, n (\%) } \\
\hline $7-17$ & $8(14.6)$ & $2(8.0)$ & $6(20.0)$ & $0.001 \ddagger$ \\
\hline $18-29$ & $13(23.6)$ & $2(8.0)$ & $11(36.7)$ & \\
\hline $30-39$ & $17(30.9)$ & $7(28.0)$ & $10(33.3)$ & \\
\hline $40-66$ & $17(30.9)$ & $14(56.0)$ & $3(10.0)$ & \\
\hline Sex, n (\%) & $n=56$ & $n=25$ & $\mathrm{n}=31$ & \\
\hline Male & $38(67.9)$ & $17(68.0)$ & $21(67.7)$ & $>0.999 \S$ \\
\hline Female & $18(32.1)$ & $8(32.0)$ & $10(32.3)$ & \\
\hline Occupation, n (\%) & $n=46$ & $n=25$ & $n=21$ & \\
\hline Unemployed & $1(2.2)$ & $1(4.0)$ & $0(0.0)$ & $0.012 \ddagger$ \\
\hline Housewife & $4(8.7)$ & $3(12.0)$ & $1(4.8)$ & \\
\hline Peasant farmer & $2(4.4)$ & $0(0.0)$ & $2(9.5)$ & \\
\hline Business & $17(37.0)$ & $9(36.0)$ & $8(38.1)$ & \\
\hline Armed forces & $2(4.4)$ & $2(8.0)$ & $0(0.0)$ & \\
\hline Services and sales & $4(8.7)$ & $4(16.0)$ & $0(0.0)$ & \\
\hline Professionals & $2(4.4)$ & $2(8.0)$ & $0(0.0)$ & \\
\hline Technicians & $1(2.2)$ & $1(4.0)$ & $0(0.0)$ & \\
\hline Other & $13(28.3)$ & $3(12.0)$ & $10(47.6)$ & \\
\hline Smoking status, $\mathrm{n}(\%)$ & $n=56$ & $\mathrm{n}=25$ & $n=31$ & \\
\hline Never/unknown & $55(98.2)$ & $25(100)$ & $30(96.8)$ & $>0.999 \ddagger$ \\
\hline Former/current & $1(1.8)$ & $0(0.0)$ & $1(3.2)$ & \\
\hline Symptoms, n (\%) & $n=56$ & $n=25$ & $n=31$ & \\
\hline $\begin{array}{l}\text { Any (fever, cough, shortness of } \\
\text { breath, rhinorrhea, fatigue) }\end{array}$ & $24(42.9)$ & $11(44.0)$ & $13(41.9)$ & $0.877 \S$ \\
\hline Fever & $12(21.4)$ & $4(16.0)$ & $8(26.8)$ & $0.516 \ddagger$ \\
\hline Cough & $11(19.6)$ & $8(32.0)$ & $3(9.7)$ & $0.048 \ddagger$ \\
\hline Shortness of breath & $0(0.0)$ & $0(0.0)$ & $0(0.0)$ & १ \\
\hline Muscle ache & $4(7.1)$ & $4(16.0)$ & $0(0.0)$ & $0.034 \ddagger$ \\
\hline Confusion & $0(0.0)$ & $0(0.0)$ & $0(0.0)$ & ๆ \\
\hline Headache & $7(12.5)$ & $3(12.0)$ & $4(12.9)$ & $>0.999 \ddagger$ \\
\hline Sore throat & $3(5.4)$ & $1(4.0)$ & $2(6.5)$ & $>0.999 \ddagger$ \\
\hline Rhinorrhea & $9(16.1)$ & $6(24.0)$ & $3(9.7)$ & $0.272 \ddagger$ \\
\hline Chest pain & $2(3.6)$ & $0(0.0)$ & $2(6.5)$ & $0.497 \ddagger$ \\
\hline Diarrhoea & $2(3.6)$ & $1(4.0)$ & $1(3.2)$ & $>0.999 \ddagger$ \\
\hline Nausea and vomiting & $2(3.6)$ & $1(4.0)$ & $1(3.2)$ & $>0.999 \ddagger$ \\
\hline Cough with sputum production & $3(5.4)$ & $2(8.0)$ & $1(3.2)$ & $0.581 \ddagger$ \\
\hline Cough with haemoptysis & $0(0.0)$ & $0(0.0)$ & $0(0.0)$ & П \\
\hline Wheezing & $0(0.0)$ & $0(0.0)$ & $0(0.0)$ & ๆ \\
\hline Joint pain & $2(3.6)$ & $2(8.0)$ & $0(0.0)$ & $0.195 \ddagger$ \\
\hline Fatigue/malaise & $4(7.1)$ & $4(16.0)$ & $0(0.0)$ & $0.034 \ddagger$ \\
\hline
\end{tabular}




\begin{tabular}{|c|c|c|c|c|}
\hline Characteristic & All & $\begin{array}{l}\text { Mulago National } \\
\text { Specialised Hospital }\end{array}$ & $\begin{array}{l}\text { Entebbe Regional } \\
\text { Referral Hospital }\end{array}$ & $P$ value \\
\hline Inability to walk & $0(0.0)$ & $0(0.0)$ & $0(0.0)$ & ๆ \\
\hline Lower chest pain & $0(0.0)$ & $0(0.0)$ & $0(0.0)$ & ๆ \\
\hline Seizures & $0(0.0)$ & $0(0.0)$ & $0(0.0)$ & ๆ \\
\hline Abdominal pain & $3(5.4)$ & $1(4.0)$ & $2(6.5)$ & $>0.999 \ddagger$ \\
\hline Conjunctivitis & $1(1.8)$ & $1(4.0)$ & $0(0.0)$ & $0.446 \ddagger$ \\
\hline Skin rash & $1(1.8)$ & $1(4.0)$ & $0(0.0)$ & $0.446 \ddagger$ \\
\hline Skin ulcers & $0(0.0)$ & $0(0.0)$ & $0(0.0)$ & १ \\
\hline Lymphadenopathy & $0(0.0)$ & $0(0.0)$ & $0(0.0)$ & १ \\
\hline Bleeding & $0(0.0)$ & $0(0.0)$ & $0(0.0)$ & १ \\
\hline General weakness & $0(0.0)$ & $0(0.0)$ & $0(0.0)$ & ๆ \\
\hline Inability to feed & $0(0.0)$ & $0(0.0)$ & $0(0.0)$ & ๆ \\
\hline Reduced activity & $0(0.0)$ & $0(0.0)$ & $0(0.0)$ & ๆ \\
\hline Pain on swallowing & $0(0.0)$ & $0(0.0)$ & $0(0.0)$ & ๆ \\
\hline Others & $0(0.0)$ & $0(0.0)$ & $0(0.0)$ & ๆ \\
\hline \multicolumn{5}{|l|}{ Vital signs } \\
\hline Temperature $\left({ }^{\circ} \mathrm{C}\right)$ & $\mathrm{n}=56$ & $\mathrm{n}=25$ & $n=31$ & \\
\hline Mean (SD) & $36.5(0.81)$ & $36.5(0.94)$ & $36.5(0.71)$ & $0.763^{*}$ \\
\hline Median (IQR) & $36.4(36.2-36.6)$ & $36.5(36.3-36.8)$ & $36.4(36.2-36.5)$ & $0.252 \dagger$ \\
\hline \multicolumn{5}{|l|}{ Categorical } \\
\hline$<37.5$ & $51(91.1)$ & $23(92.0)$ & $28(90.3)$ & $>0.999 \ddagger$ \\
\hline $37.5-38.0$ & $2(3.6)$ & $1(4.0)$ & $1(3.2)$ & \\
\hline $38.1-39.0$ & $3(5.4)$ & $1(4.0)$ & $2(6.5)$ & \\
\hline$>39.0$ & $0(0.0)$ & $0(0.0)$ & $0(0.0)$ & \\
\hline $\mathrm{SPO}_{2}$ & $\mathrm{n}=51$ & $\mathrm{n}=20$ & $n=31$ & \\
\hline Mean (SD) & $97.8(1.2)$ & $97.9(1.4)$ & $97.7(1.1)$ & $0.755^{*}$ \\
\hline Median (IQR) & 98 (97-99) & 98 (97-99) & 98 (97-99) & $0.697 \dagger$ \\
\hline$<93, \mathrm{n}(\%)$ & $0(0.0)$ & $0(0.0)$ & $0(0.0)$ & П \\
\hline Pulse rate & $n=54$ & $n=24$ & $n=29$ & \\
\hline Mean (SD) & $86.9(13.0)$ & $86.8(14.3)$ & $87.0(12.0)$ & $0.956^{\star}$ \\
\hline Median (IQR) & 87 (78-95) & $86(78-91)$ & $88(78-96)$ & $0.690 \dagger$ \\
\hline Blood pressure $(\mathrm{mm} \mathrm{Hg}), \mathrm{n}(\%)$ & $\mathrm{n}=54$ & $\mathrm{n}=24$ & $n=30$ & \\
\hline$>130 / 90$ & $20(37.0)$ & $13(54.2)$ & $7(23.3)$ & $0.020 \S$ \\
\hline$>140 / 90$ & $15(27.8)$ & $9(37.5)$ & $6(20.0)$ & $0.154 \S$ \\
\hline Body Mass Index & $n=19$ & $\mathrm{n}=19$ & $\mathrm{n}=0$ & \\
\hline Mean (SD) & $28.3(6.2)$ & $28.3(6.2)$ & & ๆ \\
\hline Median (IQR) & $27.2(23.1-32.0)$ & $27.2(23.1-32.0)$ & & ๆ \\
\hline$<18.5$ & $0(0.0)$ & $0(0.0)$ & & १ \\
\hline $18.5-24.9$ & $7(36.8)$ & $7(36.8)$ & & \\
\hline 25-29.9 & $5(26.3)$ & $5(26.3)$ & & \\
\hline $30+$ & $7(36.8)$ & $7(36.8)$ & & \\
\hline
\end{tabular}

${ }^{*} \mathrm{t}$-Test.

†Wilcoxon rank-sum test.

¥Fisher's exact test.

$\S \chi^{2}$ test.

Ilnestimable.

Bold $\mathrm{p}$ values, Statistically significant; $\mathrm{SpO}_{2}$, saturation of peripheral oxygen. 
(a)

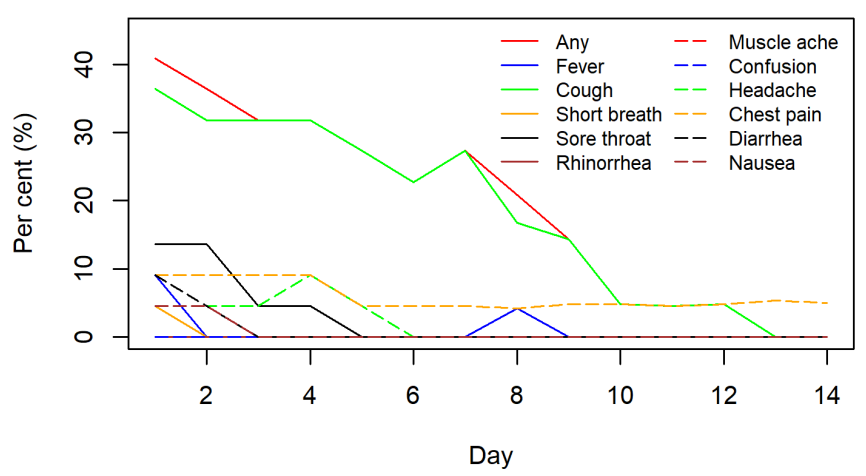

(c)

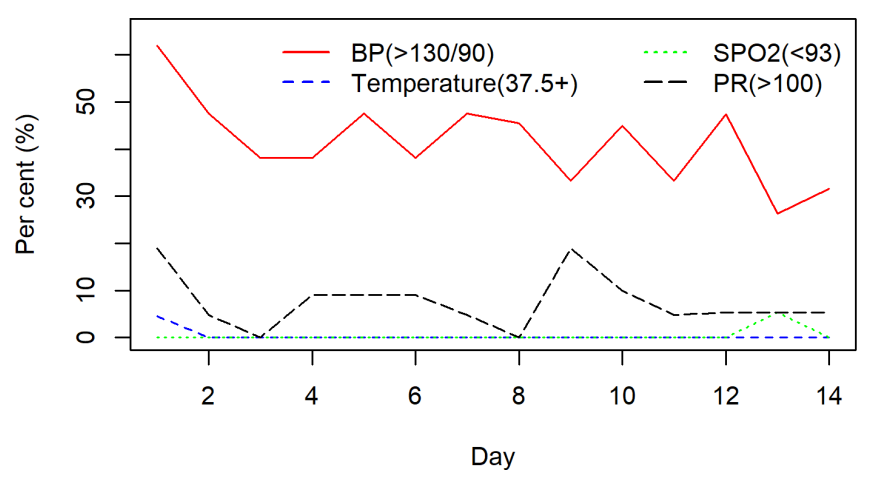

(b)

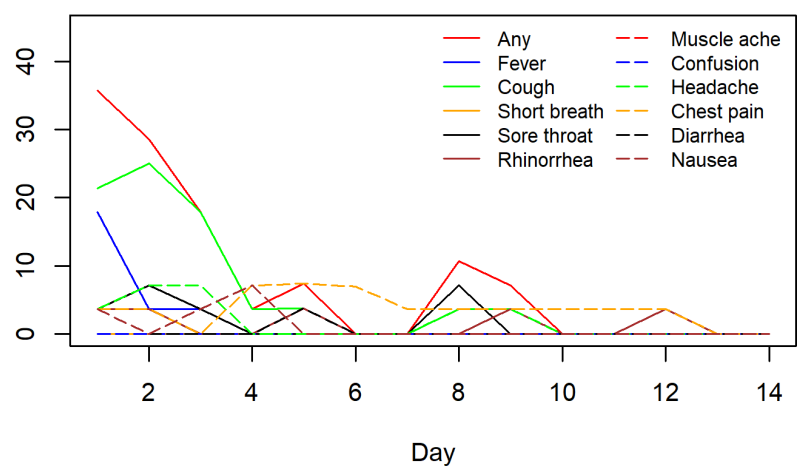

(d)

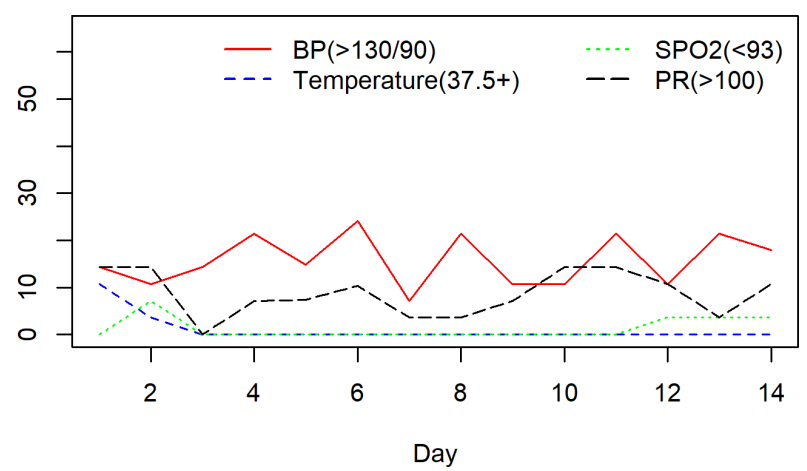

Day

Figure 1 Percentage distribution of symptoms (top row) and vital statistics (bottom row) observed among patients with COVID-19 over 14 days stratified by sites: Mulago $(A, C)$ and Entebbe (B,D). BP, blood pressure; PR, pulse rate ; $\mathrm{SpO}_{2}$, saturation of peripheral oxygen.

breath. A comparison of sociodemographic, clinical and laboratory parameters assessed in the study did not find statistical differences between asymptomatic and symptomatic patients (online supplemental table 1).

Up to $26.8 \%$ of the patients reported at least one comorbidity (any of tuberculosis, HIV, hypertension, diabetes, asthma, COPD, cancer, chronic kidney disease, chronic liver disease and chronic neurological disorders). The most common comorbidities were hypertension $(10.7 \%)$, diabetes $(10.7 \%)$, cardiovascular disease other than hypertension $(8.9 \%)$ and HIV $(7.1 \%)$. We obtained Body Mass Index (BMI) from 19 patients. The median BMI was 27.2 (IQR 23.1-32.0). Seven of these patients $(36.6 \%)$ had a BMI of more than $30,5(26.3 \%)$ had BMI 25.0-29.9, 7 (36.8\%), 18.5-24.9, and no patient had a BMI of less than 18.5.

\section{Vital signs}

Five patients $(8.9 \%)$ had a temperature of $\geq 37.5^{\circ} \mathrm{C}$; 20 patients $(37.0 \%)$ had a BP of $>130 / 90 \mathrm{~mm} \mathrm{Hg}$ at baseline; Mulago National Specialised Hospital versus Entebbe Regional Referral Hospital $(54.2 \%$ vs $23.3 \%$, $\mathrm{p}=0.020)$ while 15 patients $(27.8 \%)$ were found to have BP $>140 / 90 \mathrm{mmHg}$. Data on daily symptoms and vital signs (BP, temperature, $\mathrm{PR}$ and $\mathrm{SpO}_{2}$ ) were available for the first for 51 patients. The trajectory of these symptoms and signs over the 14 days is illustrated in figure 1. Most of the symptoms had resolved by day 10, although the cough persisted until day 12. At the Mulago National Specialised Hospital site, there was a peak in symptoms at around day 4 and another one around day 8 . At the Entebbe Regional Referral Hospital site, the trajectory of symptoms had four identifiable peaks: around day 2, 5, 8 and 12. Many patients had high BPs at baseline (note only $6(10.7 \%)$ without a prior diagnosis of hypertension). High BPs continued throughout the 14 days; higher at the Mulago National Specialised Hospital site than Entebbe Regional Referral Hospital site (figure 1C,D).

\section{Laboratory, electrocardiograph and imaging findings}

Logistical constraints precluded performance of some key COVID-19 care laboratory tests. The results of tests that were performed are shown in table 2. Five patients $(10.6 \%)$ had leucopenia (total white cell count $\leq 4000 / \mathrm{mL})^{3}$; four of these five were from the Mulago National Specialised Hospital site. Other laboratory abnormalities were as follows: lymphopenia $11.1 \%$, thrombocytopenia $26.3 \%$, AST $>40 \mathrm{U} / \mathrm{L} 8.5 \%$, ALT $>40 \mathrm{U} / \mathrm{L} 12.8 \%$ and total bilirubin $>17.1 \mu \mathrm{mol} / \mathrm{L} 13.3 \%$. Electrocardiograph was performed on 32 patients. All electrocardiograph results were normal 
Table 2 Laboratory findings among patients infected with SARS-CoV-2 admitted to two hospitals in Uganda

\begin{tabular}{|c|c|c|c|c|}
\hline Characteristic & All & $\begin{array}{l}\text { Mulago National } \\
\text { Specialised } \\
\text { Hospital/-HCQ }\end{array}$ & $\begin{array}{l}\text { Entebbe Regional } \\
\text { Referral Hospital/+HCQ }\end{array}$ & $P$ value \\
\hline \multicolumn{5}{|l|}{ Laboratory } \\
\hline White cell count $/ \mathrm{mm}^{3}$ & $\mathrm{n}=47$ & $\mathrm{n}=21$ & $\mathrm{n}=26$ & \\
\hline Median (IQR) & $5.1(4.2-6.2)$ & $5.0(4.2-6.3)$ & $5.1(4.5-6.1)$ & $0.473^{\star}$ \\
\hline \multicolumn{5}{|l|}{ Distribution, n (\%) } \\
\hline$\geq 10000 / \mathrm{mm}^{3}$ & $0(0.0)$ & $0(0.0)$ & $0(0.0)$ & \\
\hline $4001-<10000 / \mathrm{mm}^{3}$ & $42(89.4)$ & $17(81.0)$ & $25(92.1)$ & $0.158 \dagger$ \\
\hline$\leq 4000 / \mathrm{mm}^{3}$ & $5(10.6)$ & $4(19.0)$ & $1(3.9)$ & \\
\hline Neutrophil count & $\mathrm{n}=45$ & $\mathrm{n}=19$ & $n=26$ & \\
\hline Median (IQR) $/ \mathrm{mm}^{3}$ & $2(2-2)$ & $2(1-2)$ & $2(2-2)$ & $0.092^{*}$ \\
\hline \multicolumn{5}{|l|}{ Distribution, n (\%) } \\
\hline$\leq 1500 / \mathrm{mm}^{3}$ & $7(15.6)$ & $5(26.3)$ & $2(7.7)$ & $0.114 \dagger$ \\
\hline Lymphocyte count & $\mathrm{n}=45$ & $\mathrm{n}=19$ & $n=26$ & \\
\hline Median (IQR)/mm³ & $2.3(1.8-2.9)$ & $2.4(1.4-2.9)$ & $2.3(2.0-2.9)$ & $0.340^{*}$ \\
\hline \multicolumn{5}{|l|}{ Distribution, n (\%) } \\
\hline$\leq 1500 / \mathrm{mm}^{3}$ & $5(11.1)$ & $5(26.3)$ & $0(0.0)$ & $0.010 \dagger$ \\
\hline Platelets per $\mathrm{mm}^{3}$ & $n=19$ & $\mathrm{n}=18$ & $\mathrm{n}=1$ & \\
\hline Median (IQR) & 227 (121-275) & $224.5(121-275)$ & 232 & $0.855^{\star}$ \\
\hline \multicolumn{5}{|l|}{ Distribution, n (\%) } \\
\hline$<150000 / \mathrm{mm}^{3}$ & $5(26.3)$ & $5(27.8)$ & $0(0.0)$ & $>0.999 \dagger$ \\
\hline Haemoglobin (g/L) & $\mathrm{n}=19$ & $\mathrm{n}=18$ & $n=1$ & \\
\hline Median (IQR) & $1.47(1.34-1.60)$ & $1.47(1.34-1.57)$ & 1.62 & $0.273^{*}$ \\
\hline \multicolumn{5}{|l|}{ Distribution, n (\%) } \\
\hline$\leq 1.0 \mathrm{~g} / \mathrm{L}$ & $2(10.5)$ & $2(11.1)$ & $0(0.0)$ & $>0.999 \dagger$ \\
\hline Aspartate aminotransferase $>40 \mathrm{U} / \mathrm{L}, \mathrm{n} / \mathrm{N}(\%)$ & $4 / 47(8.5)$ & $3 / 18(16.7)$ & $1 / 29(3.5)$ & $0.150 \dagger$ \\
\hline Alanine aminotransferase $>40 \mathrm{U} / \mathrm{L}, \mathrm{n} / \mathrm{N}(\%)$ & 6/47 (12.8) & $5 / 18(27.8)$ & $1 / 29(3.5)$ & $0.025 \dagger$ \\
\hline $\mathrm{LDH} \geq 250 \mathrm{U} / \mathrm{L}, \mathrm{n} / \mathrm{N}(\%)$ lactate & $0 / 11(0.0)$ & $0 / 11(0.0)$ & & \\
\hline Creatinine $\geq 133 \mu \mathrm{mol} / \mathrm{L}, \mathrm{n} / \mathrm{N}(\%)$ & $0 / 48(0.0)$ & $0 / 19(0.0)$ & $0 / 29(0.0)$ & - \\
\hline Troponin, median (IQR) & $0.1(0.1-0.1)$ & $0.1(0.1-0.1)$ & $0.1(0.1-0.1)$ & $0.532^{*}$ \\
\hline Total bilirubin $>17.1 \mu \mathrm{mol} / \mathrm{L}, \mathrm{n} / \mathrm{N}(\%)$ & 2/15 (13.3) & 2/14 (14.3) & $0 / 1(0.0)$ & $>0.999 \dagger$ \\
\hline Total bilirubin, median (IQR) & $2.5(0-12.9)$ & $2.4(0-11.7)$ & 13.8 & $0.232^{*}$ \\
\hline $\mathrm{C}$ reactive protein $\geq 10 \mathrm{mg} / \mathrm{L}, \mathrm{n} / \mathrm{N}(\%)$ & 6/49 (12.2) & $5 / 24(20.8)$ & $1 / 25(4.0)$ & $0.098 \dagger$ \\
\hline Median (IQR) & $1.7(0.5-8.1)$ & $4.0(1.8-9.7)$ & $0.5(0.5-1.4)$ & $<0.001^{*}$ \\
\hline Procalcitonin ng/mL & $n=49$ & $n=24$ & $n=25$ & \\
\hline Median (IQR) & $0.1(0.1-0.12)$ & $0.1(0.1-0.12)$ & $0.1(0.1-0.13)$ & $0.614^{*}$ \\
\hline Lactate dehydrogenase & $n=49$ & $n=24$ & $\mathrm{n}=25$ & \\
\hline Median (IQR) & $330(287-402)$ & $318(244-385)$ & 330.5 (295.5-402.5) & $0.282 \dagger$ \\
\hline
\end{tabular}

*Wilcoxon rank-sum test.

†Fisher's exact test.

LDH, lactate dehydrogenase.

with the exception of bradycardia and first-degree heart block in a 66-year patient who was being treated for hypertension with atenolol before admission. We performed CXRs on 21 patients, three of which had abnormal results consisting of bilateral infiltrates (two patients) and unilateral right lower zone infiltrates (one patient). We performed a CT scan on one patient to further evaluate reasons for persistent clinical and radiological features noted from the preliminary investigations.

Treatments and clinical outcomes

Data on treatments provided to the patients are presented in table 3. Patients at the Entebbe Regional 
Table 3 Treatments and clinical outcomes of patients infected with SARS-CoV-2 during the first 2 weeks of admission at Mulago National Specialised Hospital and Entebbe Regional Referral Hospital

\begin{tabular}{lllll}
\hline Variable & All & $\begin{array}{l}\text { Mulago National } \\
\text { Specialised Hospital }\end{array}$ & $\begin{array}{l}\text { Entebbe Regional } \\
\text { Referral Hospital }\end{array}$ & P value \\
\hline Treatments & $\mathrm{n}=55$ & $\mathrm{n}=24$ & $\mathrm{n}=31$ & \\
$\quad$ Hydroxychloroquine, $\mathrm{n}(\%)$ & $29(52.7)$ & $0(0.0)$ & $29(93.6)$ & $<0.001^{*}$ \\
\hline $\begin{array}{l}\text { Antibiotics, } \mathrm{n}(\%) \\
\text { Clinical outcomes at data cut-off, no (\%) }\end{array}$ & $42(76.4)$ & $12(50.0)$ & $30(96.8)$ & $<0.001^{*}$ \\
\hline $\begin{array}{l}\text { Length of hospitalisation (days) } \\
\quad \text { Mean (SD) }\end{array}$ & $\mathrm{n}=47$ & $\mathrm{n}=20$ & $\mathrm{n}=27$ & \\
$\quad$ Median (IQR) & $20.6(4.1)$ & $19.3(1.5)$ & $21.5(5.1)$ & $0.068 \dagger$ \\
\hline $\begin{array}{l}\text { Discharged from hospital with two negative RT- } \\
\text { PCRs }\end{array}$ & $20(19-21)$ & $19(18-20)$ & $21(19-22)$ & $0.012 \ddagger$ \\
\hline Death & 56 & 25 & 31 & \\
\hline Admitted to intensive care unit & 0 & & & \\
\hline
\end{tabular}

*Fisher's exact test.

tt-Test.

$\ddagger$ Wilcoxon rank-sum test.

RT-PCR, reverse transcriptase PCR.

Referral Hospital site received HCQ (29), while all the 24 patients at the Mulago National Specialised Hospital site did not receive HCQ. Patients were treated with a range of antibiotics including azithromycin, ampicillin/ cloxacillin combination and Augmentin. The average antibiotic prescription rate was $76.4 \%$. The primary outcomes (admission to ICU, mechanical ventilation or death) did not occur in any patient. The average length of hospitalisation was 20.6 days. The time to clinical recovery (TTCR), defined as the time to clearance of the major COVID-19 symptoms, is shown in figure 2. TTCR was earlier among patients treated with HCQ (6.4 days) and those who did not ( 8.6 days), but this difference did not reach statistical significance $(\mathrm{p}=0.204$, figure $2 \mathrm{~A})$ We monitored the patients for known HCQ side effects among those treated with or without HCQ. The rates of these events are presented in online supplemental table 2. Although the number of patients is small, the rates of these events did not seem to be significantly higher among HCQ-treated patients.
A

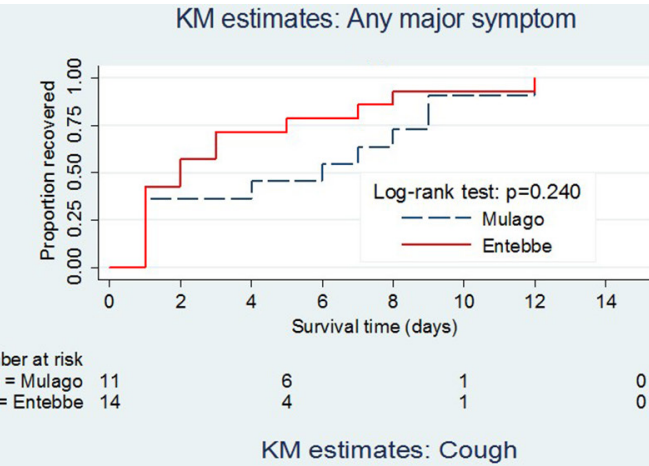

C

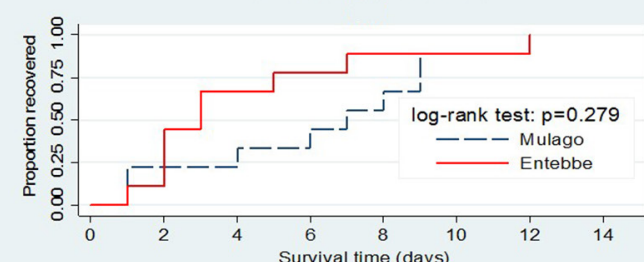

Number at risk

site $=$ Entebo 9

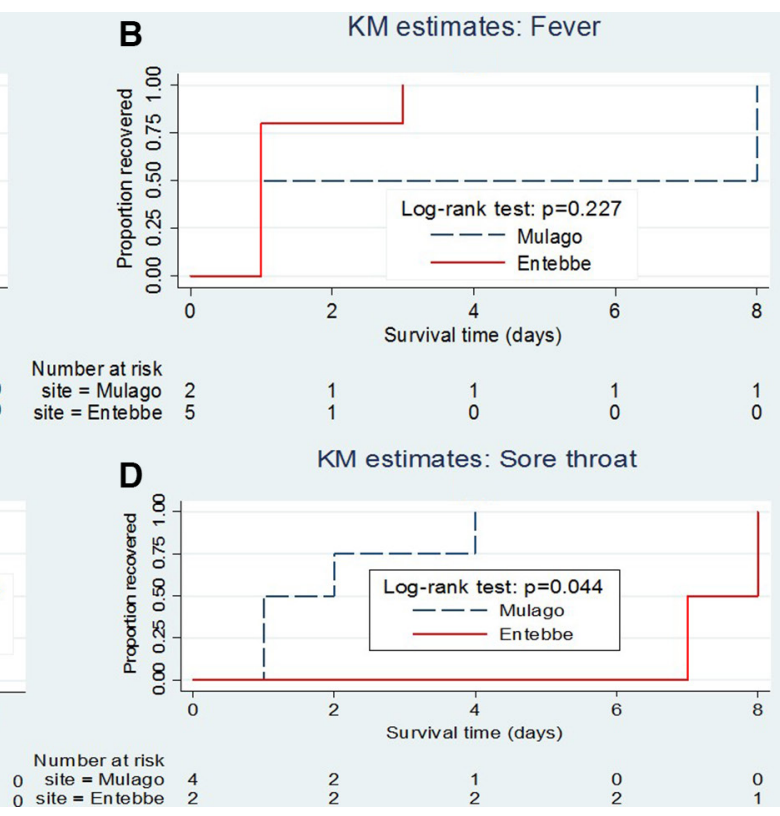

Figure 2 KM curves showing proportion of symptomatic patients with COVID-19 19 recovering from various symptoms; any major symptom (top left), fever (top right), cough (bottom left) and sore throat (bottom right) over 14 days of follow-up. Major symptoms include fever, cough, sore throat, rhinorrhea and shortness of breath. KM, Kaplan-Meier. 


\section{DISCUSSION}

In this prospective study, we describe the characteristics and outcomes of 56 treated patients with COVID-19 at two hospitals in Uganda. The median age of these patients was 33 years, and $67.9 \%$ of the patients were male. The predominance of male gender among patients with COVID-19 is similar to what has been reported elsewhere. ${ }^{7}{ }^{17}$ Like most respiratory diseases COVID-19 predominance in men may reflect exposure dynamics since men are usually more outdoor and more likely to be exposed than women. The mean age we observed is, however, different from reports in China, Europe and the USA. ${ }^{17}$ In China, Guan $e t a l^{7}$ reported a mean age of 47 years, while in the USA, Richardson $e a^{18}$ reported a mean age of 63 years. The young age of the Ugandan patients with COVID-19 is probably a reflection of the general population structure where our population is composed mainly of young people. ${ }^{19}$ Another reason could be the source of the initial patients with COVID-19 in Uganda, which was mainly composed of youthful travellers returning from their workplaces abroad. We also note that the 56 patients include eight children belonging to a choir who were returning from abroad. This could also have skewed the cohort's age downwards.

We found that up to $56.1 \%$ of the patients were asymptomatic and not in keeping with most studies on hospitalised patients. In most studies, over $80 \%$ of the patients have one or more COVID-19 symptoms by the time of hospitalisation. ${ }^{4717}$ Our results are, however, in line with findings from a study by Arons $e t a l^{20}$ and Sutton $e t$ $a l^{5}$ In the study by Arons et $a l^{20}$ among 89 residents of a skilled nursing home, $56 \%$ of persons found positive were asymptomatic. ${ }^{20}$ The testing in this study was part of a survey. In the case of Uganda, most of the persons were tested as part of routine surveillance because they either had travelled or had been in contact with a confirmed case. It seems therefore that the absence of symptoms could be due to early detection. In the study by Arons $e t$ $a l^{20}$ referred to earlier, they reassessed the asymptomatic persons again. They found that 24 of the 27 asymptomatic patients subsequently developed symptoms (median time to onset of 4 days). This was not the case among our patients, suggesting that there could be other reasons for lack of symptoms in our cohort. ${ }^{20}$ Asymptomatic disease has gained attention following the finding that disease transmission occurs even in the absence of symptoms. ${ }^{21}$ If indeed most patients with COVID-19 in Uganda and Africa will be asymptomatic, control efforts need to be geared towards mass testing as many of those infected will not be sick and will therefore not be presenting at health facilities for testing and treatment.

Comorbidity has been a common finding in patients with COVID-19 and has been found to be associated with worse outcomes. ${ }^{41722}$ In this study, we found that up to $26.8 \%$ of the patients reported at least one comorbidity (any of tuberculosis, HIV, hypertension, diabetes, asthma, COPD, cancer, chronic kidney disease, chronic liver disease or chronic neurological disorders). The most common comorbidities were hypertension (10.7\%), diabetes $(10.7 \%)$, cardiovascular disease other than hypertension $(8.9 \%)$ and HIV $(7.1 \%)$. The rate of having any of the listed comorbidities and the individual comorbidity rates are much lower than reported elsewhere. ${ }^{717}$ The lower rates are probably a reflection of lower rates of the same diseases in the general population but could also be due to lower rates of diagnosis of these diseases in the general population. A case in point is hypertension; only six patients reported a history of hypertension. However, as can be seen in table 1, $37 \%$ of the patients had $\mathrm{BP}>130 / 90 \mathrm{~mm} \mathrm{Hg}$, and $27.8 \%$ had BP higher than $140 / 90 \mathrm{~mm} \mathrm{Hg}$. Living with undiagnosed hypertension has been reported in several studies in Uganda. ${ }^{23} \mathrm{~A}$ study by Kayima et $a l^{23}$ found that among 553 hypertensive persons, only $13.7 \%$ were aware of their diagnosis. The underdiagnosis notwithstanding, 37\% prevalence of hypertension in these patients is higher than that found in general population studies in Uganda. ${ }^{23-25}$ Anxiety due to the fear of the disease could explain this high baseline BP. Further research is needed to establish if there is a direct link between SARS-CoV-2 virus infection and high BP, especially through the hypothalamic-pituitary-adrenal axis.

The rates of laboratory derangements investigated were lower than those reported in cohorts elsewhere, in keeping with the mild nature of the disease in the patients we studied. ${ }^{7}$ For example, we found leucopenia in $10.6 \%$ of the patients, but Guan et al found it at $33.7 \%$ among Chinese patients; lymphopenia was $11.1 \%$ compared with $80.4 \%$ found in China. The same applied to CXR abnormalities where we found that only 3 out of 21 patients who underwent this examination had abnormalities. In the Guan $e t a l^{7}$ study, this rate was $59.1 \%$.

In this initial cohort of 56 patients, no death, admission to ICU or mechanical ventilation was observed. With the exception of two patients, almost all had mild disease at admission and none deteriorated to severe or critical disease, a clinical trajectory that has not been observed in other countries. This patient, a 40-year-old man, was admitted with RT-PCR-confirmed COVID-19. He had been previously diagnosed with hypertension treated with calcium channel blockers and a thiazide diuretic. He had a week-long cough and during his stay, low $\mathrm{SpO}_{2}$ ranging between $92 \%$ and $95 \%$ was noted. His baseline CXR (2 April) showed bilateral infiltrates (figure 3A and follow-up CXRs (6 and 17 April, respectively; figure 3B,C). The 17 April CXR showed marked improvement. On 19 April before discharge, we performed chest CT scans on this patient. The scan showed multiple peripheral and subpleural GGOs with associated interlobular septal thickening in all segments of the lung parenchyma. There was coalescing with a tendency to form air space consolidation. Selected slices from the chest CT scans are shown (figure 3). Although CXR had shown marked improvement, a CT scan performed later showed presence of pathology (figure 4). 

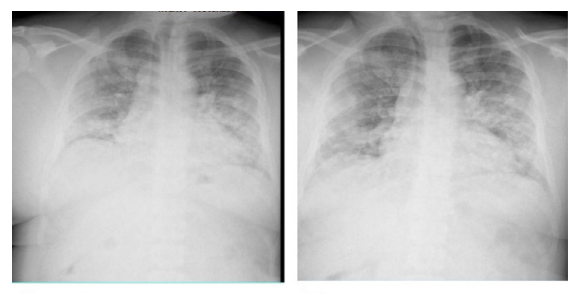

B

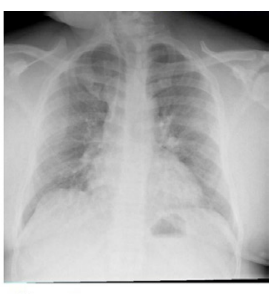

c

Figure 3 Posteroanterior chest X-ray images of a 40-yearold male hypertensive Ugandan admitted with reverse transcriptase PCR confirmed COVID-19 at baseline (A), 4 days $(B)$ and 15 days $(C)$ postadmission at Mulago National Specialised Hospital.

Death, admission to ICU and mechanical ventilation were observed at rates of $21 \%$ death, $14.2 \%$ admission to ICU and 3.2\% mechanical ventilation in a study involving 5700 Americans by Richardson et al. ${ }^{18}$ In China, the rates were $6.1 \%$ for death, $5 \%$ for admission to ICU and $2.3 \%$ for mechanical ventilation. ${ }^{7}$ The reasons for the lack of these adverse outcomes are not clear to us, but we think it could be due to the small sample size. It could also be due to the mild nature of the disease, with the reasons for the disease being mild not known at this point in time. The low rates of comorbid disease are probably a key factor since these have been consistently found to be associated with adverse outcomes. ${ }^{78}{ }^{22}$ Several studies suggest that the viral load patients with COVID-19 exhibit is linked to the initial dose of the virus at the time of infection, and the viral load in turn determines the severity of the disease, although most of such studies are small and retrospective. ${ }^{2627}$ Most of the Ugandan patients were detected among travellers whose exposures during travel could have been lower, hence low viral load at the beginning of infection and hence mild disease. Further research is needed if this similar trend continues to be observed.

Most of the patients in this cohort received supportive care and many received antibiotics. The main driving factor for the high antibiotic use is the entrenched practice of prescribing antibiotics for respiratory symptoms in our setting. In addition, there is evidence that secondary bacterial infection occurs following respiratory viral infection. When they occur, they are usually due to Streptococcus pneumonia, Haemophilus influenza, and Staphylococcus aureus. ${ }^{28}$ However, secondary bacterial infection in COVID-19 has been reported to be rare. ${ }^{18}$ In view of this finding that secondary bacterial infections are uncommon in COVID-19 as opposed to other viral pneumonias, we do not recommend routine use of antibiotics in the treatment of COVID19. A comparison of 29 patients who received HCQ to 24 patients who did not received HCQ showed that there was no difference in terms of mortality, admission to ICU, mechanical ventilation and TTCR. TTCR was shorter for the HCQ group, but it did not reach statistical significance. HCQ is prescribed in many centres around the world for the treatment of COVID-19. HCQ was reported to result in improved viral clearance in a small study done in France. ${ }^{12} \mathrm{~A}$ recently rapid review has identified 38 studies on the use of HCQ for treatment of COVID-19 (8 randomised clinical trials and 30 observational studies) ${ }^{29}$ This review notes the low methodological quality of the identified studies but concludes that the available evidence is sufficient to conclude that HCQ has no benefit in the treatment and prevention of COVID-19. ${ }^{29}$ Our findings, although from a small
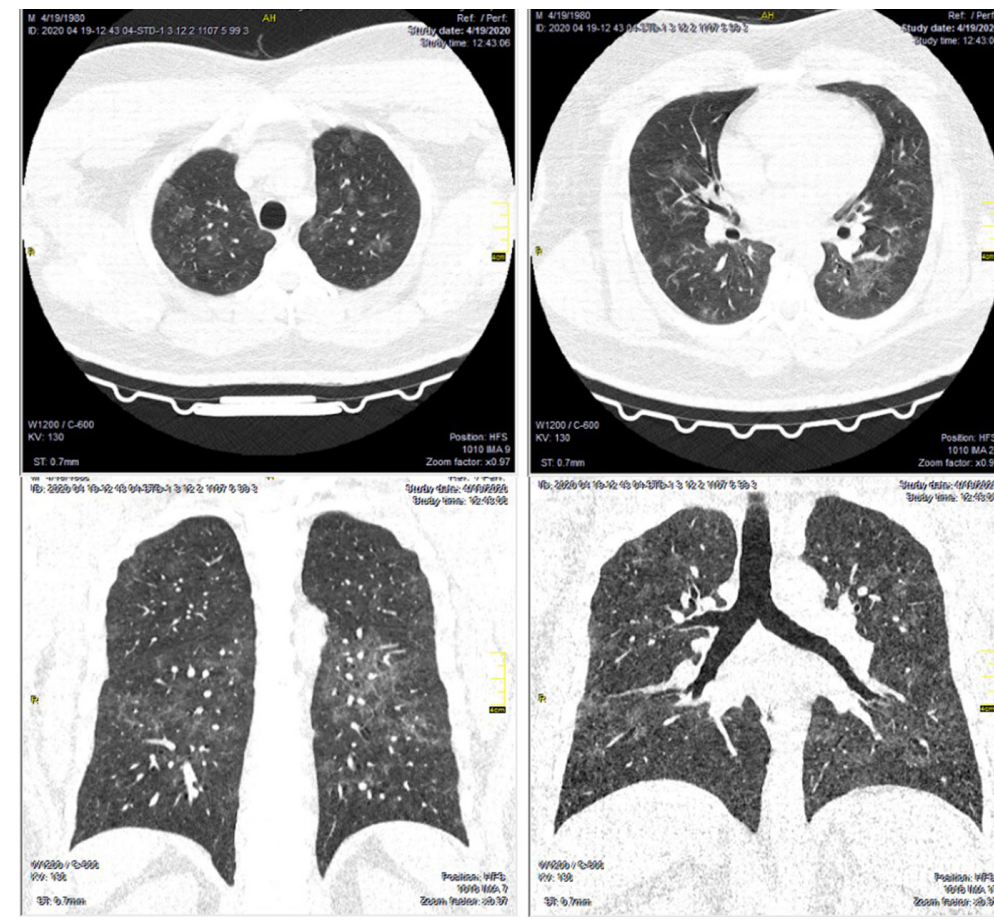
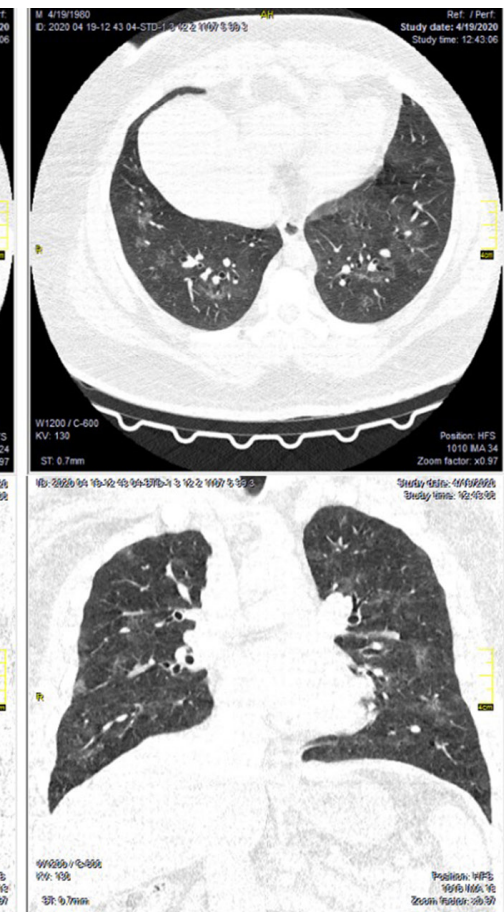

Figure 4 CT scan images of the 40-year-old male Ugandan patient with COVID-19 at discharge (17 days postadmission). 
observation study, are in line with studies included in this review.

Our study has limitations, including the small sample size, and limitation of tests, including absence of viral clearance monitoring. The outcomes by HCQ are also limited by lack of randomisation to HCQ treatment.

In conclusion, most of the patients with COVID-19 presented with mild disease and exhibited a clinical trajectory not similar to other countries. Outcomes did not differ by HCQ treatment status in line with other concluded studies on the use of HCQ in the treatment of COVID-19.

\section{Author affiliations}

${ }^{1}$ Lung Institute, Makerere University, Kampala, Uganda

${ }^{2}$ Dpartment of Medicine, Entebbe Regional Referral Hospital, Entebbe, Uganda ${ }^{3}$ Pulmonary and Critical Care, Johns Hopkins University, Baltimore, Maryland, USA

${ }^{4}$ Uganda Peoples Defense Forces, Uganda Heart Institute Ltd, Kampala, Uganda

${ }^{5}$ Department of Radiology, Mulago National Specialised Hospital, Kampala, Uganda

${ }^{6}$ Uganda Ministry of Health, Kampala, Uganda

${ }^{7}$ Department of Medicine, Mulago National Specialised Hospital, Kampala, Uganda

${ }^{8}$ Department of Immunology and Molecular Biology, Makerere University College of Health Sciences, Kampala, Uganda

${ }^{9}$ Department of Paediatrics, Mulago National Specialised Hospital, Kampala Uganda

${ }^{10}$ Department of Nursing, Mulago National Specialised Hospital, Kampala, Uganda

${ }^{11}$ School of Public Health, Makerere University College of Health Sciences, Kampala, Uganda

${ }^{12}$ Department of Medicine, Entebbe Regional Referral Hospital, Entebbe, Uganda

${ }^{13}$ Pathology, Makerere University College of Health Sciences, Kampala, Uganda

${ }^{14}$ Medicine, Makerere University College of Health Sciences, Kampala, Uganda

${ }^{15}$ Republic of Uganda Ministry of Health, Kampala, Uganda

${ }^{16}$ Entebbe Regional Refferal Hospital, Makerere University, Kampala, Uganda

Acknowledgements We thank the patients for their participation in the study. We extend our thanks to the health workers who collected the data, the data officers and all administrative staff for their contribution to the implementation of this project, and also patient advisors and counsellors for their contribution to the implementation of this project.

Contributors Conception and design: BK, WM, AK, WW, MLJ, NF, RKB and WB. Analysis and interpretation: LM, RS and NK. Drafting the manuscript for important intellectual content: BK, AK, WM, RN, IK, WK, BSB, EN, HA-T, BA, TS, MM, SK, MLJ, DK, BB, MRK, HM, CN, JL, RKB, EB and WB.

Funding This study was sponsored and funded by the Makerere University Research and Innovation Funds, Kampala Uganda.

Competing interests None declared.

Patient and public involvement Patients and/or the public were not involved in the design, conduct, reporting or dissemination plans of this research.

Patient consent for publication Not required.

Ethics approval This study was conducted according to the principles of the Declaration of Helsinki and good clinical practice (GCP) as laid down by the ICH topic E6 (Note for Guidance on GCP). The study was approved by the Mulago Hospital Research and Ethics Committee (MHREC 1869). All patients provided written informed consent.

Provenance and peer review Not commissioned; externally peer reviewed.

Data availability statement Data are available at Makerere University Lung Institute upon reasonable request. Please contact Dr Bruce Kirenga for data requests.
Open access This is an open access article distributed in accordance with the Creative Commons Attribution Non Commercial (CC BY-NC 4.0) license, which permits others to distribute, remix, adapt, build upon this work non-commercially, and license their derivative works on different terms, provided the original work is properly cited, appropriate credit is given, any changes made indicated, and the use is non-commercial. See: http://creativecommons.org/licenses/by-nc/4.0/.

ORCID iD

Bruce Kirenga http://orcid.org/0000-0002-2023-2840

\section{REFERENCES}

1 Zhu N, Zhang D, Wang W, et al. A novel coronavirus from patients with pneumonia in China, 2019. N Engl J Med 2020;382:727-33.

2 World Health Organization. Coronavirus disease (COVID-19) pandemic. Available: https://www.who.int/emergencies/diseases/ novel-coronavirus-2019 [Accessed 21 Apr 2020].

3 World Health Organization. COVID-19 in the WHO African Region. Available: https://www.afro.who.int/health-topics/coronavirus-covid19 [Accessed 21 Apr 2020].

4 Huang C, Wang Y, Li X, et al. Clinical features of patients infected with 2019 novel coronavirus in Wuhan, China. Lancet 2020;395:497-506

5 Sutton D, Fuchs K, D'Alton M, et al. Universal screening for SARS-CoV-2 in women admitted for delivery. N Engl J Med 2020;382:2163-4.

6 Liang W-H, Guan W-J, Li C-C, et al. Clinical characteristics and outcomes of hospitalised patients with COVID-19 treated in Hubei (epicentre) and outside Hubei (non-epicentre): a nationwide analysis of China. Eur Respir J 2020;55:2000562.

7 Guan W-J, Ni Z-Y, Hu Y, et al. Clinical characteristics of coronavirus disease 2019 in China. N Engl J Med 2020;382:1708-20.

8 Report of the who-china joint mission on coronavirus disease 2019 (covid-19) 2020;8.

9 Phua J, Weng L, Ling L, et al. Intensive care management of coronavirus disease 2019 (COVID-19): challenges and recommendations. Lancet Respir Med 2020;8:506-17.

10 Chen C, Huang J, Cheng Z, et al. Favipiravir versus arbidol for COVID-19: a randomized clinical trial. medRxiv 2020.

11 Caly L, Druce JD, Catton MG, et al. The FDA-approved drug ivermectin inhibits the replication of SARS-CoV-2 in vitro. Antiviral Res 2020;178:104787.

12 Gautret P, Lagier J-C, Parola P, et al. Hydroxychloroquine and azithromycin as a treatment of COVID-19: results of an openlabel non-randomized clinical trial. Int J Antimicrob Agents 2020;56:105949.

13 Grein J, Ohmagari N, Shin D, et al. Compassionate use of Remdesivir for patients with severe Covid-19. N Engl J Med 2020;382:2327-36.

14 Xu X, Han M, Li T, et al. Effective treatment of severe COVID-19 patients with tocilizumab. Proc Natl Acad Sci U S A 2020;117:10970-5.

15 Duan K, Liu B, Li C, et al. Effectiveness of convalescent plasma therapy in severe COVID-19 patients. Proc Natl Acad Sci U S A 2020;117:9490-6.

16 Coronavirus disease (COVID-19) technical guidance: patient management. Available: https://www.who.int/emergencies/diseases/ novel-coronavirus-2019/technical-guidance/patient-management [Accessed 22 Apr 2020].

17 Garg S, Kim L, Whitaker M, et al. Hospitalization rates and characteristics of patients hospitalized with laboratory-confirmed coronavirus disease 2019 - COVID-NET, 14 States, March 1-30, 2020. MMWR Morb Mortal Wkly Rep 2020;69:458-64.

18 Richardson S, Hirsch JS, Narasimhan M, et al. Presenting characteristics, comorbidities, and outcomes among 5700 patients hospitalized with COVID-19 in the New York City area. JAMA 2020;323:2052

19 Uganda Bureau of Statistics. The National population and housing census 2014-main report, 2016.

20 Arons MM, Hatfield KM, Reddy SC, et al. Presymptomatic SARSCoV-2 infections and transmission in a skilled nursing facility. $N$ Engl $J$ Med 2020;382:2081-90.

21 Wei WE, Li Z, Chiew CJ, et al. Presymptomatic Transmission of SARS-CoV-2 - Singapore, January 23-March 16, 2020. MMWR Morb Mortal Wkly Rep 2020;69:411-5.

22 Mehra MR, Desai SS, Kuy S, et al. Cardiovascular disease, drug therapy, and mortality in Covid-19. N Engl J Med 2020;382:e102.

23 Kayima J, Nankabirwa J, Sinabulya I, et al. Determinants of hypertension in a young adult Ugandan population in 
epidemiological transition-the MEPI-CVD survey. BMC Public Health 2015;15:830.

24 Kwarisiima D, Balzer L, Heller D, et al. Population-based assessment of hypertension epidemiology and risk factors among HIV-positive and general populations in rural Uganda. PLoS One 2016;11:e0156309.

25 Lunyera J, Kirenga B, Stanifer JW, et al. Geographic differences in the prevalence of hypertension in Uganda: results of a national epidemiological study. PLoS One 2018;13:e0201001.
26 Heneghan C, Brassey J. SARS-CoV-2 viral load and the severity of COVID-19. CEBM, 2020.

27 Chu C-M, Cheng VCC, Hung IFN, et al. Viral load distribution in SARS outbreak. Emerg Infect Dis 2005;11:1882-6.

28 Morris DE, Cleary DW, Clarke SC. Secondary bacterial infections associated with influenza pandemics. Front Microbiol 2017;8:1041.

29 Ongoing living update of potential COVID-19 therapeutics: summary of rapid systematic reviews, 2020. Available: https://iris.paho.org/ handle/10665.2/52294 [Accessed 31 Jul 2020]. 